\title{
Changing Abundances, Changing Opacities
}

\author{
Jason W. Ferguson ${ }^{1}$, and Aaron Dotter ${ }^{2}$ \\ ${ }^{1}$ Department of Physics, Wichita State University, Wichita, KS 67260-0032, USA \\ email: jason.ferguson@wichita.edu \\ ${ }^{2}$ Department of Physics and Astronomy, Dartmouth College, 6127 Wilder Laboratory, \\ Hanover, NH 03755, USA \\ email:aaron.l.dotter@dartmouth.edu
}

\begin{abstract}
With ever changing solar abundances being reported the equation of state and opacities needed for stellar evolution models also change. A discussion of those changes in mean molecular opacities will be presented with a discussion on the effect on evolution models. Aside from changing the abundances of the base mixture the enrichment changes too. Traditionally mean opacity tables are produced for oxygen-rich mixtures, however stars will often become carbon-rich. A discussion of carbon-rich opacities tables will also be presented.
\end{abstract}

Keywords. atomic processes, equation of state, molecular processes, stars: abundances, stars: evolution

\section{Background}

For the caluculation of the transfer of radiation in a stellar evolution model or any astrophysical environment, it is necessary to understand and include the interaction of radiation with matter. The physics of attenuation in a medium is called the opacity of material and is a function of the properties of the material (temperature, density or pressure, chemical composition, etc.) and a function of the wavelength of light. Computation of opacities require detailed knowledge of the atomic, molecular, and dust physics over a wide range of wavelengths. A technique that is often used to simplify the computation of radiative transfer is to take a wavelength averaged or mean opacity.

Tables of mean opacities as functions of temperature and density have been compiled for decades (see Iglesias \& Rogers(1991) and Iglesias \& Rogers(1996), OPAL hereafter; Seaton et al.(1994), OP hereafter; Alexander \& Ferguson(1994) and Ferguson et al.(2005), F05 hereafter). The advantage of a grey opacity is the savings of hours of computation time. For example, in a stellar evolution model the age of the star must be advanced in small timesteps at certain stages in the star's evolution and the additional time sink of computing the detailed opacity with the evolution is currently computationally impossible in a reasonable time. The disadvantage of using mean opacity tables is the loss of chemical composition changes for which opacity tables are not available and the closest "set" has to be used. As outlined in F05 the typical mean opacity table set includes over $155 \mathrm{X}$ and $\mathrm{Z}$ number fractions each with a large range of temperature and density. A full set can take more than four days of computer time on a small cluster.

This review summarizes the recent work in low temperature (less than $10,000 \mathrm{~K}$ ) opacities at Wichita State University (WSU). A summary of input physics is discussed first, followed by a section on how new solar abundances mixtures affect the opacity and the effect of changing $\mathrm{C} / \mathrm{O}$ ratios on the mean opacity. The effect of changing elemental abundances on stellar evolution models is discussed and the last section looks at future directions. 


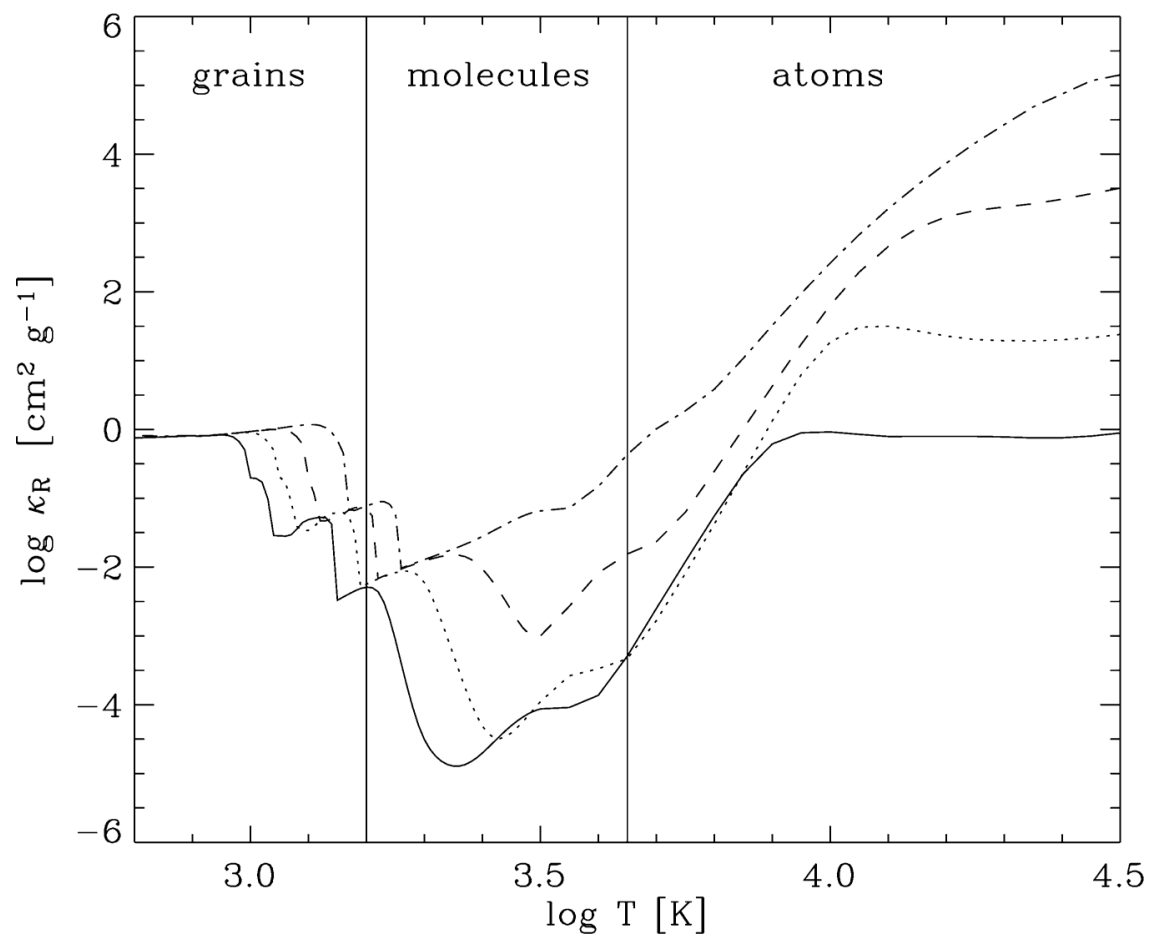

Figure 1. A plot of mean opacity as a function of temperature for four values of $\log R$. From bottom to top the solid line is for a $\log R=-5.0$, then is $\log R=-3.0$ (dotted), $\log R=-1.0$ (dashed), and $\log R=+1.0$ (dash-dot) with the highest mean opacity.

\section{Input Physics}

A thourogh discussion of the input physics included in WSU opacity tables is given in F05. Figure 1 shows what the Rosseland mean opacity looks like as a function of temperature for the typical quasi-solar mixture $(\mathrm{X}=0.7$ and $\mathrm{Z}=0.02)$ with four densities included. Density is often parameterized as $R$ which is definded as $\rho / T_{6}^{3}$ with the temperature in millions of Kelvin.

The Rosseland mean is defined as

$$
\frac{1}{\kappa_{R}} \equiv \frac{\int_{0}^{\infty} \frac{1}{\kappa_{\lambda}} \frac{\partial B_{\lambda}}{\partial T} d \lambda}{\int_{0}^{\infty} \frac{\partial B_{\lambda}}{\partial T} d \lambda}
$$

where $\kappa_{\lambda}$ is the monochromatic opacity, $B_{\lambda}$ is the Planck function and $\partial B_{\lambda} / \partial T$ is therefore the weighting function of the Rosseland mean. For the computations from F05, we integrate Eq. 1 over 24,000 wavelengths. The wavelengths are not evenly spaced and the details are given in F05. What is important to note is that the Rosseland mean is a harmonic mean, not a straight mean. With a straight mean additional sources can effectively be added to the total opacity, but with a harmonic mean it is necessary to compute $\kappa_{\lambda}$ for each different or new composition that might occur.

For illustration purposes the dominate phase of material that controls the strength of the mean opacity is indicated in the figure. Note that the dividing lines are approximate and move in temperature with density, which is espcecially evident that the molecular/grain line at about $1500 \mathrm{~K}$. 


\subsection{Atoms}

Atoms, including free-free and bound-free sources, dominate the mean opacity at temperatures above about $4,000 \mathrm{~K}$ as shown in Fig. 1. As the temperature of the gas decreases the opacity also decreases as the atoms become more neutral and the electrons tend to settle in the lowest levels. The most important opacity sources include the most abundant species, hydrogen and helium. Hydrogen lines are very important for temperatures above $\sim 10,000 \mathrm{~K}$. The continuous source $\mathrm{H}^{-}$is important as the temperature falls below 10,000 K. F05 includes some discussion showing the mean opacity with some sources removed to see the overall importance of them.

For higher values of gas density $(\log R)$ the mean opacity is generally larger. This is due primarily to pressure ionization and collision effects of the atoms and pressure broadening of the lines. However, it is not the rule. For example, between about 4,000 K and $10,000 \mathrm{~K}$ the mean opacity of the the $\log R=-3$ is lower than the mean opacity of $\log R=-5$. This effect is due to more $\mathrm{H}^{-}$opacity at the lower density.

\subsection{Molecules}

As a gas cools molecules form under equilibrium conditions. This is reflected in the mean opacity in two ways. In Fig. 1 at the higher end of the "molecules" region is a bump in the mean opacity that is due to the beginning formation of strongly bonded molecules such as CO. While the abundance is quite small at these temperatures there are enough $\mathrm{CO}$ lines to begin to affect the mean opacity in small ways.

At even lower temperatures the mean opacity rises as $\mathrm{H}_{2} \mathrm{O}$ and $\mathrm{TiO}$ begin to appear. Both of these molecules, especially water, have huge numbers of lines in the region of the weighting function of the Rosseland mean that amplifies the effect they have on the opacity. The rise in the mean opacity can be as much as 2 orders of magnitude depending upon the gas density peaks, and then begins to fall away again as the temperature lowers and the rotational/vibrational modes begin to settle down.

Higher gas densities result in larger values of the mean opacity. In general the water bump appears washed out due to higher gas density. The peak of the water bump moves to higher temperature with increasing density as well.

\subsection{Dust}

At even lower tempreatures the gas can condense and solid precipitates exist in equilibrium which greatly affect the mean opacity. In general the first condensate to form is either $\mathrm{CaTiO}_{3}$ or $\mathrm{Al}_{2} \mathrm{O}_{3}$ and the mean opacity jumps by an order of magnitude in a very small temperature range. Other species then form, including $\mathrm{MgSiO}_{3}, \mathrm{FeMgSiO}_{4}$ and $\mathrm{Fe}$ among others, and begin to generate a mean opacity that is roughly constant with temperature.

A larger value of gas density moves the condensation temperature of the grains. This is evident in Fig. 1 as the line between the grains/molecule regions shifts towards higher temperature. Once the grains exist at lower temperatures the mean opacity is roughly constant.

It should be noted that all of the opacities shown in this paper come from the routines discussed in F05 and include the assumptions of dust physics outlined in that paper. Ferguson et al. (2007) discusses the affects of the basic grain physics assumptions (including size distribution, porosity, aggregates, etc.) on the mean opacity. In general these effects are not currently needed for the computation of stellar evolution models as models today do not fall below the grain condensation temperature. 


\section{Abundance Effects}

In light of recent changes in the metallicity mixture of the sun, especially the amount of solar oxygen, an illustration of how these changes affect the mean opacity is given in Figure 2. In the top portion of the figure the mean opacity is given for the abundance mixtures of Grevesse \& Noels (1993 GN93 hereafter ), Grevesse \& Sauval (1998; GS98 hereafter ), Seaton (private communication; S92 hereafter), Asplund et al. (2005; AGS05 hereafter ) and Lodders (2003; L03 hereafter ) for the same scaled abundance set (X=0.7 and $\mathrm{Z}=0.02$ ).

In the lower portion of the figure the differences in the logarithm of the opacity is shown indicating how much of a true change abundances have on the mean opacity. The GN93 mixture is used as a baseline in the plot for illustration purposes. At higher temperatures where the mean opacity is due mostly to hydrogen the difference between the five abundance sets is neglibible. However, at cooler temperatures when molecules become important the differences become quite large. At temperatures where water is the dominate opacity, differences can be as large for as $25 \%$ for the difference between AGS05 and GN93. This is sensible because the AGS05 abundances have an decrease in the amount of oxygen and the amount of water is also less.

At even cooler temperatures the differences in the opacities are generally due to the refractory elements. It is clear from Fig. 1 that the temperature of the condensation of grains move a bit in temperature for the different sets by observing the discontinuities in the lower portion of the figure.

Another feature in opacity calculations that is needed by stellar evolution modellers is the effect of $\alpha$-element enhancement. Figure 3 shows the effect of a typical alpha enhancement based on the abundances of S92. The $\alpha$-element abundances are from Achim Weiss (private communication; S92ae hereafter) and clearly show the effect of more metals, particularly oxygen. At the higher temperatures where hydrogen controls the opacity the difference between the GN93 and the $\alpha$-enhanced mix is nearly neglible as there is a bit more atomic opacity in the S92ae mix compared with the comparison mixture.

There are clear differences in the water bump at about $\log T \sim 3.25$ as much more oxygen is available and there is more water in the s92ae mix compared with the GN93 mix. At cooler temperatures the dust opacity is actually lower due to the scaling of the total metallicity $Z$, which is kept constant for both s92ae and GN93.

\subsection{Changing the $\mathrm{C} / \mathrm{O}$ ratio}

Of the many consequences of stellar evolution one that is often overlooked in terms of opacity is the changing from an oxygen rich to a carbon rich environment. For high temperature opacities OPAL has produced tables for enhanced carbon and oxygen abundances (see Rogers \& Iglesias 1993). Known as OPAL type 2 tables there are hundreds of combinations of $X, Z, d X_{C}$ and $d X_{O}$ which have been computed. Essentially the abundance of carbon is enhanced as helium is reduced as the triple-alpha process ensues. Then, material from the star's core is then dredged up causing the outer layers to become carbon rich.

Carbon-rich opacities for low temperatures are not as readily aviaible. AF94 and F05 do not provide carbon-enhanced tables. Extrapolations based on oxygen rich opacities are avaible from Marigo (2002) and more recently tables from Lederer \& Aringer (2008) are among those tables that are publically available.

Figure 4 and Figure 5 shows the effect of raising the amount of carbon in the mix. Each of the mean opacities shown in the figures are for the scaled $(X=0.70, Z=0.02)$ set scaled from GN93, $\log R=-3.0$ and without dust grains for ease of comparison. In 


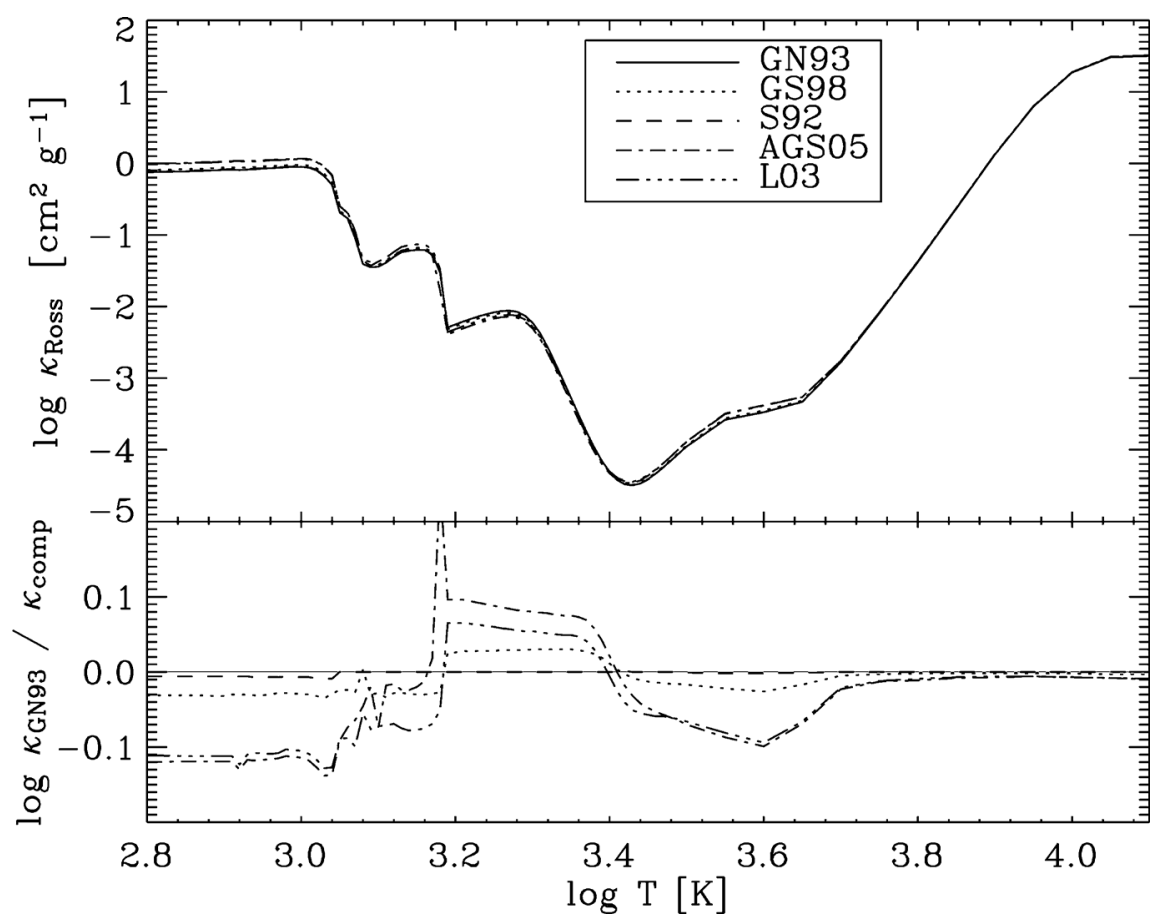

Figure 2. Shown is the mean opacity for five different abundance sets. In the top portion of the figure the solid line represents the mean opacity based on GN93 abundacnes, the dotted line from GS98, the dashed line from S92, the dash-dot line from AGS05, and the dash-dot-dot line is from L03. In the bottom portion the logarithm of the ratio of the opacity from GN93 to the mean opacity being compared to. The thin solid line is a ratio of one, the dotted line compares GN93 with GS98, the dashed line (GN93 to S92), the dash-dot line (GN93 to AGS05), and the dash-dot-dot line represents (GN93 to L03).

Fig. 4 the mean opacity for mixes with $C / O$ ratios less than 1.0 are shown. It is easy to see the water bump at $\log T \sim 3.25$ becomes smaller as the $C / O$ ratio rises to unity. What is interesting is that the value of $C / O=1.0$ is not the lowest mean opacity. The lowest value of the mean opacity is (for most temperatures) $C / O=0.98$. In Fig. 5 the mean opacity is shown for $C / O \geqslant 1.0$. The mean opacity rises at temperatures of $\log T \sim 3.3$ as molecular $\mathrm{CN}$ begins to dominate the opacity. In the last section of this paper, the validity of the carbon opacities used here are discussed.

Overall, the mean opacity from solar values to a $C / O$ ratio of unity to carbon rich lowers as water is removed from the mixture, then rises as cyanide begins to dominate. Similar plots have been made for the OPAL type 2 abundances with F05, however the $d X_{C}$ and $d X_{O}$ enhancements have such large stepsizes that most of the physics involved as the opacity changes is lost. Large stepsizes may be reasonable at higher temperatures as hydrogen and other ionized species dominate the opacity. The smaller step sizes as the $C / O$ ratio is slowly increased retains more of the changing opacity due due to the changing abundances.

\subsection{Stellar evolution models}

With current computational facilities it is not yet possible to compute an entire stellar evolution model for an entire lifetrack of a star and include the detailed opacity computation as well. This reasoning explains why opacity tables are used. Dotter et al. (2007) explores the effect of changes in the abundance of individual species on the mean opacity, 


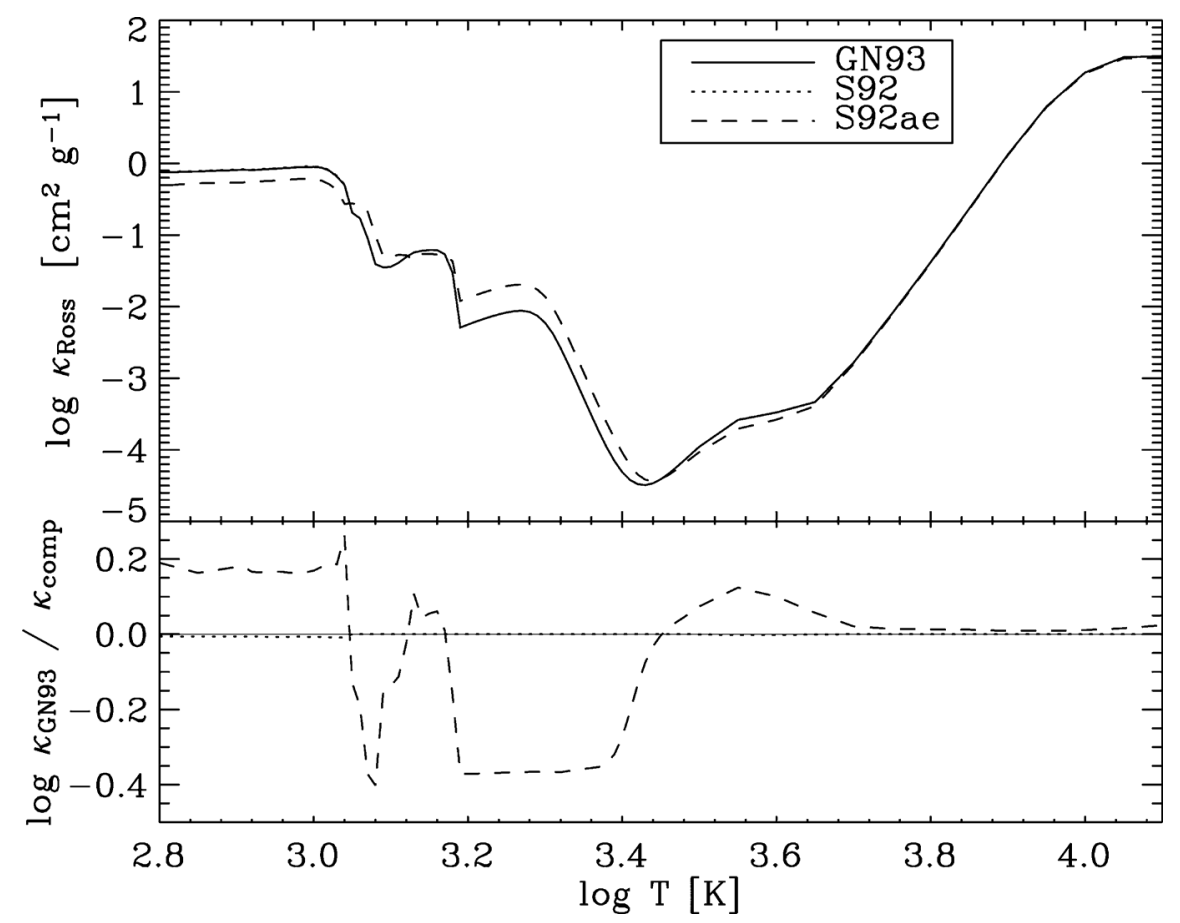

Figure 3. Similar to Fig. 1 the mean opacity is shown for GN93 abundances (solid line), S92 abundances (dotted line) and S92 with the $\alpha$-elements enhanced (dashed line). The bottom portion represents the logarithm of the ratio of the opacity from the GN93 mix compared to S92 (dotted line). The dashed line represents the ratio of the GN93 mix to S92 with $\alpha$-enhancement. Note that the scale of the lower portion is different from that shown in Fig. 1.

lifetracks of stars on the H-R diagram and on the mean main sequence lifetimes of stars as well. In that study the H-R diagram life tracks were computed for a series of mixtures each with individually enhanced elements. For example, what happens to a star if the initial fraction of $\mathrm{C}, \mathrm{N}$, or $\mathrm{O}$ is enhanced by a small fraction?

Figure 6 shows the effect of changing the abundance of individual elements on the mean opacity. Both $\mathrm{N}$ - and Ne-enhanced abundances do not have much of an effect on the mean opacity as those elements do not exist in molecules that have large numbers of lines. However, both the $\mathrm{O}$ - and $\mathrm{C}$-enhanced changes greatly affect the mean opacity, mostly in the $\log T \sim 3.3$ regime where water is an important molecular source. One noticeable feature in the figure is the rather significant change to the mean opacity caused by enhancing magnesium in the $\log T \sim 3.55$ region. This bump in the opacity is due mostly to the very broad $\mathrm{Mg}$ II line at $\lambda 2798$, the fact that $\mathrm{Mg}^{+}$is very abundadant in that temperature region, and the weighting function of the Rosseland mean opacity peak is near the Mg-line. All of these features of $\mathrm{Mg}$ help in changing the mean opacity significantly.

Stellar evolution tracks have been computed by Dotter et al. (2007) and show significant differences for individually increased elements. Figure 7 shows the effect of enhancing individual elemental abundances on $\mathrm{H}-\mathrm{R}$ digram isochrones for $\mathrm{C}$ - and O-enhanced compositions. The isochrones were computed with the Dartmouth Stellar Evolution Program (see Dotter et al. (2007) for details).

The conclusions of Dotter et al. (2007) basically boil down to the fact that low temperature opacities of various metal elements affect the lifetracks of stars in non-linear ways. 


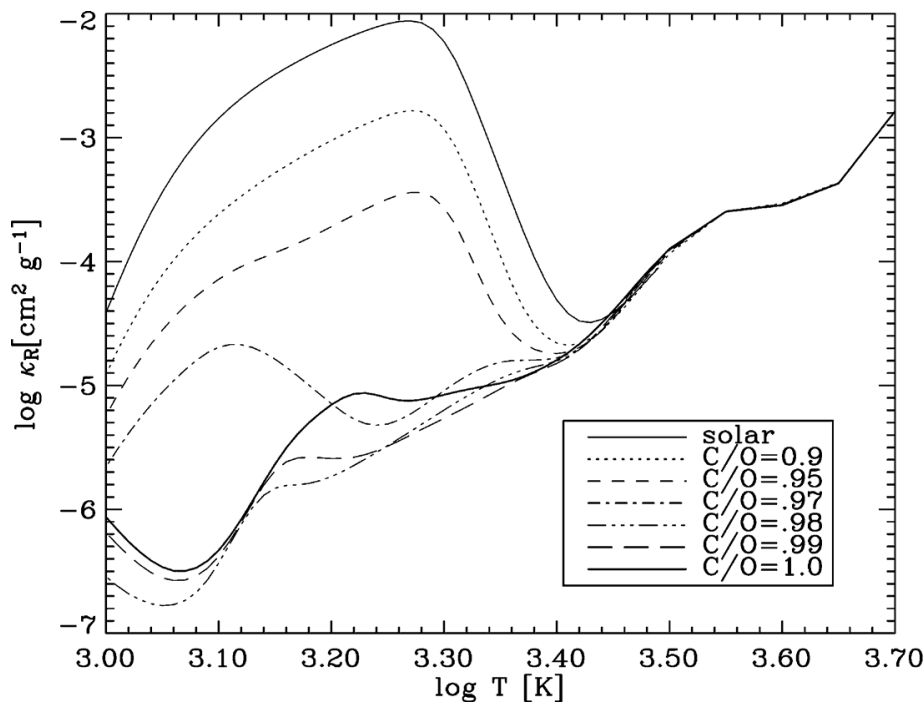

Figure 4. Shown is the mean opacity based upon calculations of varying $C / O$ ratios including a solar mixture and upto a $C / O$ ratio of unity. The legend gives the values of the $C / O$ ratio and the text discusses the details of the models.

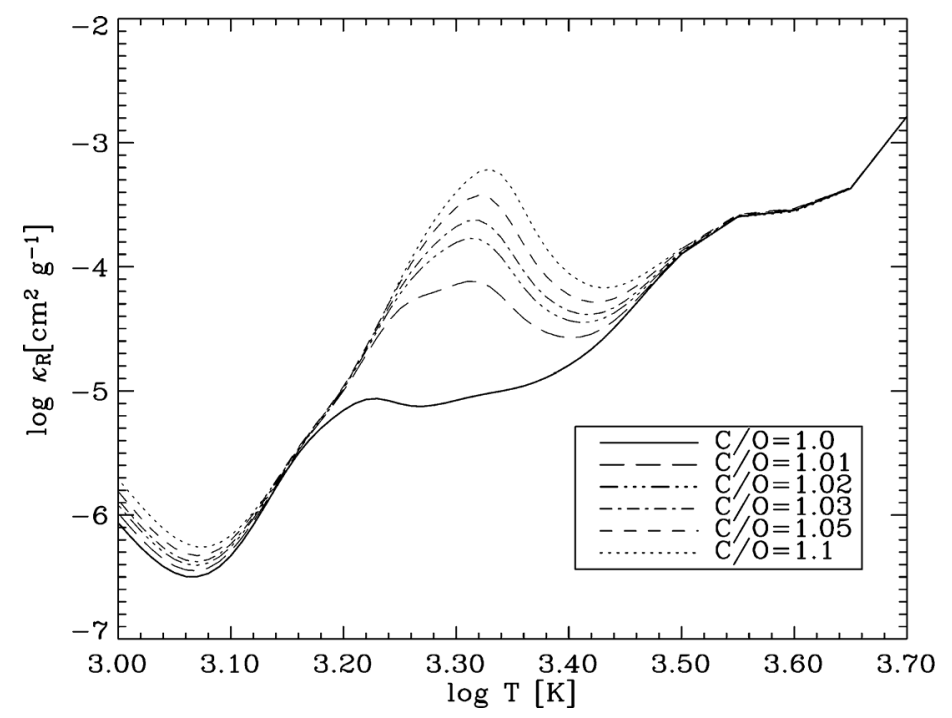

Figure 5. Similar to Fig. 4 with $C / O$ ratios greater than unity.

For example enhancing the lighter elements $(\mathrm{C}, \mathrm{N}, \mathrm{O})$ tends to lead to hotter shorter lived stars. However, enhancing heavier elements are not as easily constrained. For example, enhancing Fe tends to lead to cooler, longer lived stars, but enhancing other refractory elements lead to cooler stars, but the main sequence lifetimes vary in non-determinate ways.

Figure 8 shows the influence on main sequence lifetimes due to enhancing individual elements for four different stellar masses. Most elements only affect the lifetimes in small ways much less than $5 \%$ of the total, however a few elements, $\mathrm{O}$ and Fe, make much larger contributions. It should be noted that all of the stellar evolution results shown here are for models with constant $\mathrm{Z}$ rather than constant $[\mathrm{Fe} / \mathrm{H}]$. Enhancing an element 


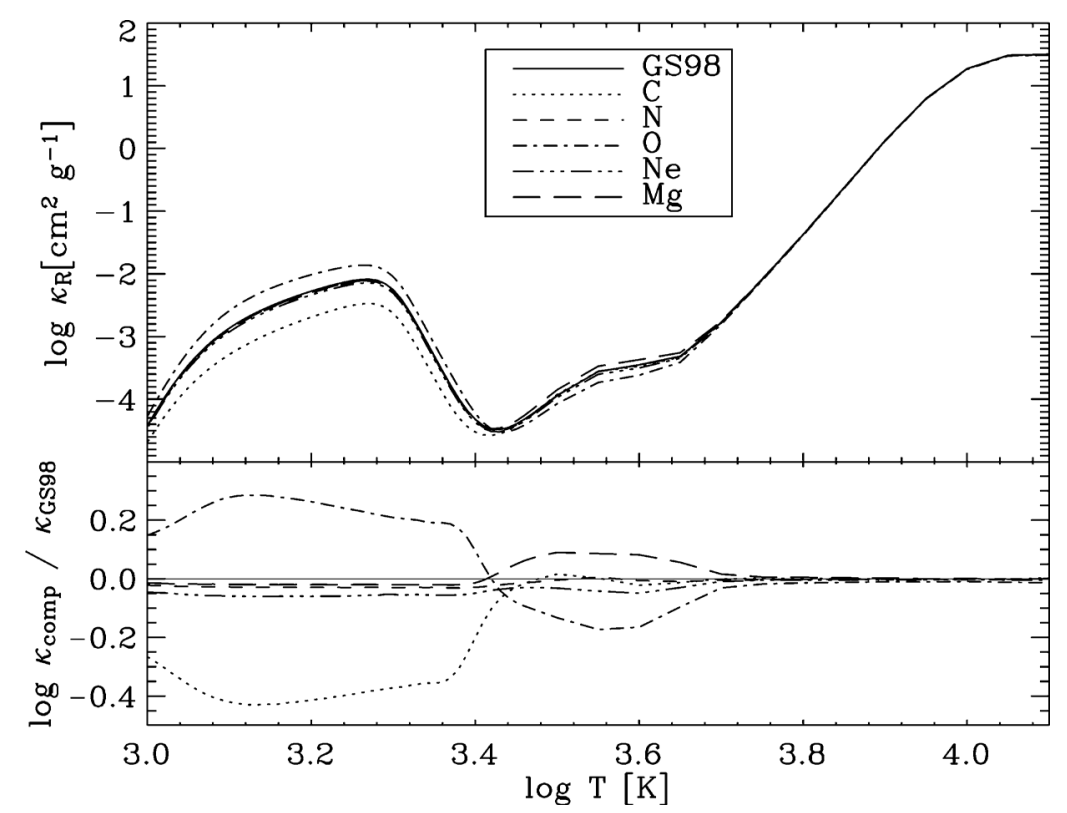

Figure 6. In the top portion of the plot are mean opacities based upon six different mixtures. The baseline consists of mean opacities computed assuming the abundance set of GS98 (solid line). Additionally mean opacities are also shown with individually enhanced abundances carbon (dotted), nitrogen (dashed), oxygen (dash-dot), neon (dash-dot-dot) and magnesium (long dash). In the bottom portion are the difference plots similar to Fig. 1. The dotted line represents the ratio of the baseline to the carbon enhanced opacities. Also shown is the GS98 to N-enhanced (dashed), GS98 to O-enchanced (dash-dot), GS98 to Ne-enhanced (dash-dot,) and GS98 to Mg-enhanced (long dash).

at constant $\mathrm{Z}$ requires other metals to be reduced. For example, as Dotter et al. (2007) points out enhancing the light elements mean less heavy elements and the opposite too: more Fe means there is now less $\mathrm{C}, \mathrm{N}$ and $\mathrm{O}$ available.

\section{Future Directions}

With regard to section 3.1 on the opacity of different values of the $C / O$ ratio there are several items that need updating in F05.

Comparisons of our carbon-rich mean opacities with those of Lederer \& Aringer (2008) shows that our opacities may be missing a some important molecular sources. Our molecular data for $\mathrm{HCN}$ and for $\mathrm{C}_{2} \mathrm{H}_{2}$ are out-of-date and do not at this time include a sufficent number of lines for the wavelength coverage necessary for the computation of mean opacities. Comparisions of PHOENIX based carbon star models with observations (Peter Woitke, private communication) show the same lack of appropriate carbon molecule coverage.

A short list of near term future needs include:

- Update carbon molecular line lists to more modern lists using the open electronic databases as outlined in Tennyson et al. (2007). The Tennyson et al. paper discusses only work done on molecules at UCL including $\mathrm{HCN} / \mathrm{HNC}, \mathrm{C}_{3}, \mathrm{H}_{3}{ }^{+}$, and HDO. Other important carbon molcules that need updating include $\mathrm{C}_{2} \mathrm{H}_{2}$ and $\mathrm{CN}$.

- Explore the effects of carbon rich grains on the mean opacity. As outlined above for the $C / O$ ratio comparisons the effects of grains were turned off in the equation of state and hence the opacity computations. Carbon grains tend to have a much higher 


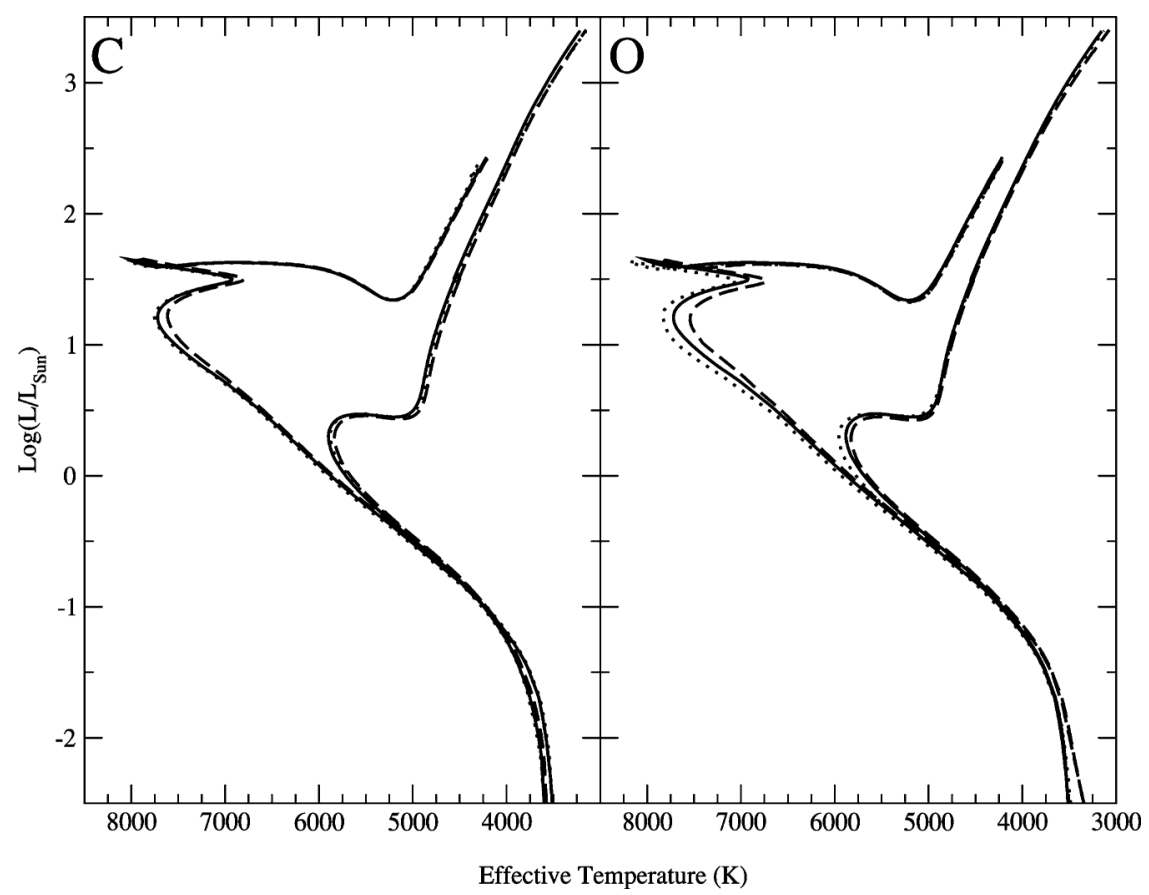

Figure 7. Plots of isochrones for 1 and 8 Gyr are shown for both C-enhanced (left) and O-enhanced (right) computations. The solid line represents the scaled solar abundance (normal computation), dotted lines are enhanced elemental abundances at constant $Z$ and dashed lines are for the enhanced abundances with constant $[\mathrm{Fe} / \mathrm{H}]$. See Dotter et al. (2007) for color versions of these plots.

mean opacity than silicate grains. The exploration of the physical properties needed to compute the opacity of carbon grains as they are very different from silicate grains will need to be accomplished as well. For example, the size distribution that is typically used in F05 is not appropriate for carbon grains which tend to be much smaller.

\section{Acknowledgements}

Low temperature astrophysics at Wichita State University is supported by NSF grant AST-0239590 with matching support from the State of Kansas. We also acknowledge the support from the National Science Foundation under Grant No. EIA-0216178 and Grant No. EPS-0236913, matching support from the State of Kansas and the Wichita State University High Performance Computing Center. JF thanks D. Alexander for many continuing fruitful discussions.

\section{References}

Alexander, D. R. \& Ferguson, J. W. 1994, ApJ, 437, 879

Asplund, M., Grevesse, N., Sauval, A. J., Allende Prieto, C., \& Blomme, R. 2005, A\&A, 431, 693

Dotter, A., Chaboyer, B., Ferguson, J. W., Lee, H.-c., Worthey, G., Jevremović, D., \& Baron, E. 2007, ApJ, 666, 403

Ferguson, J. W., Alexander, D. R., Allard, F., Barman, T., Bodnarik, J. G., Hauschildt, P. H., Heffner-Wong, A., \& Tamanai, A. 2005, ApJ, 623, 585

Ferguson, J. W., Heffner-Wong, A., Penley, J. J., Barman, T. S., \& Alexander, D. R. 2007, ApJ, 666, 261 


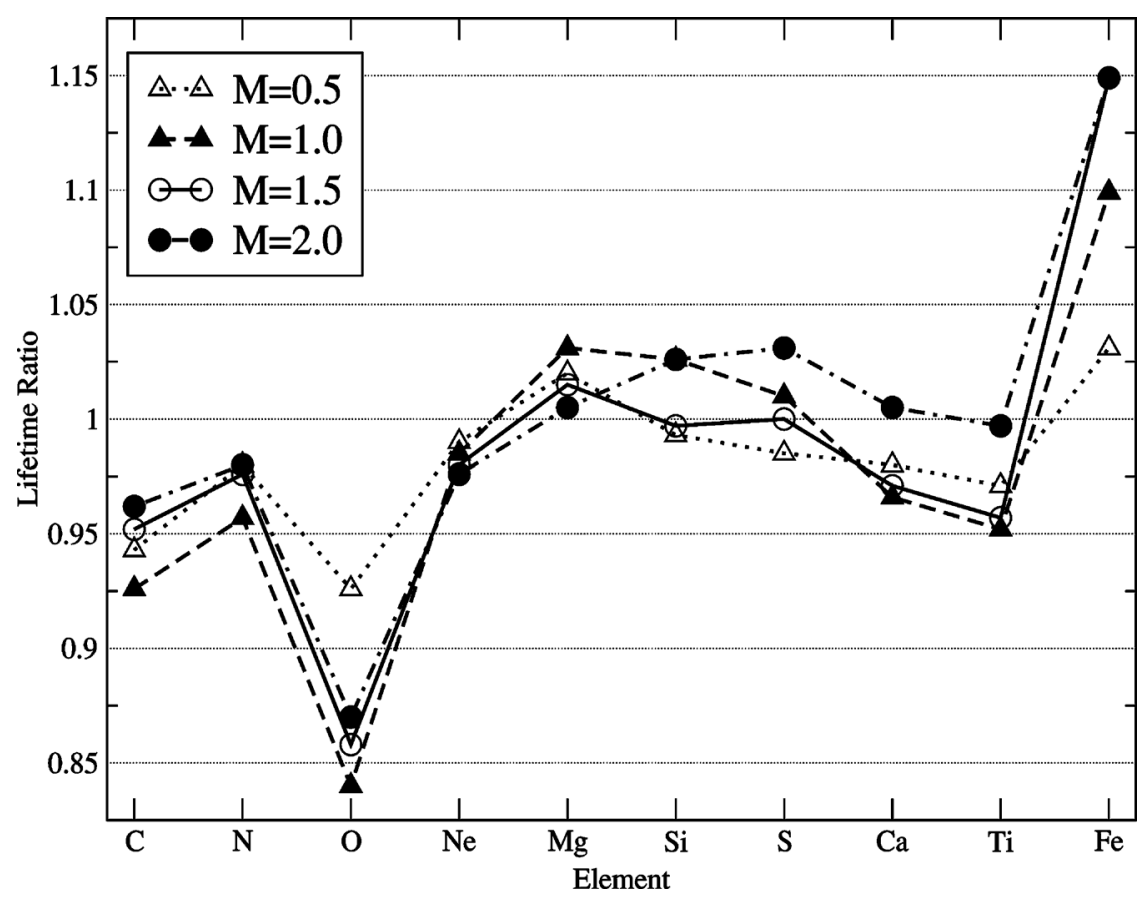

Figure 8. Plots of the ratio of main sequence lifetime due to a model with an enhanced element to a scaled-solar model to probe the effects of each element on the lifetime. Masses in the legend are given in units of solar masses and there does not appear to be any significant trend with mass. See Dotter et al. (2007) for a color version of this plot.

Grevesse, N. \& Noels, A. 1993, Origin and Evolution of the Elements

Grevesse, N. \& Sauval, A. J. 1998, Sp.Sci.Rev, 85, 161

Iglesias, C. A. \& Rogers, F. J. 1991, ApJ, 371, 408

-. 1993, ApJ, 412, 752

-. 1996, ApJ, 464, 943

Lederer, M. T. \& Aringer, B. 2008, AIPC, 1001, 11

Lodders, K. 2003, ApJ, 591, 1220

Marigo, P. 2002, A\&A, 387, 507

Seaton, M. J., Yan, Y., Mihalas, D., \& Pradhan, A. K. 1994, MNRAS, 266, 805

Tennyson, J., Harris, G. J., Barber, R. J., La Delfa, S., Voronin, B. A., Kaminsky, B. M., \& Pavlenko, Ya. V. 2007, Mol. Phys., 105, 701

\section{Discussion}

Herwig: Can you comment on molecular and dust opacities at very low $\mathrm{Z}$ with the primary contribution from AGB stars, like $\mathrm{O}, \mathrm{Al}, \mathrm{Mg}$ etc? What could be implications for mass loss?

Ferguson: Even at $Z=0.0001$ the grain opacity can be as much as $2-3$ orders of magnitude above the $Z=0$ case. If mass loss depends on radiation pressure due to grains then there may be enough opacity - but that would be environment dependent.

WILLSON: Your grain opacity is an integral over the grain distribution - what distribution did you use? 
Ferguson: The fiducial size distribution is Mathis, Rumpl + Nordsieck 1977. However, changing the distribution to another does not change the mean opacity much.

Woitke: Comment: dust size distribution $\mathrm{f}(\mathrm{a})$ matters!

- $\chi_{\lambda}$ depends strongly on $\langle a\rangle !\langle a\rangle \rightarrow \infty \Rightarrow \chi_{\lambda} \sim \frac{1}{\langle a\rangle}$ (AGB star winds $\rightarrow$ small grains; protoplanetary disks $\rightarrow$ large grains).

— in one object, $\langle a\rangle$ may depend strongly on position, e.g., brown dwarfs: $\langle a\rangle$ small in high layers $(0.01 \mu \mathrm{m}) ;\langle a\rangle$ large in deep layers $(100 \mu \mathrm{m})$.

FERGuson: Absolutely correct! And I want to point out that we can make opacity tables with (almost) any grain distribution you'd like.

Kwok: There are many observational overtone transitions of data in the visible part of the spectrum. The oscillation strengths of these highly excited lines are poorly known as hardly any laboratory data exist. Are these lines included in your opacity calculations?

FERGuson: I am not sure of this specific case. Our water list is tuned for brown dwarfs. We have $3 \mathrm{H}_{2} \mathrm{O}$ line lists and they do differ slightly in the mean opacity they produce. 